\title{
Implementation of mobile augmented reality system based on ORB-FREAK Algorithm
}

\author{
NIU Yutao,HUANG Hui \\ College of computer science and technology,Chongqing University of Posts and \\ Telecommunications, Chongqing,400065
}

12444@163.com

Keywords: Mobile augmented reality, ORB-FREAK, Hamming distance, Android.

\begin{abstract}
At present, the existing algorithm registration for mobile equipment is weak on real-time feature,this paper proposes an Tracking registration for Mobile augmented reality based on ORB-FREAK algorithm.To Reduce the algorrithm time overhead,using ORB and FREAK as detecter and descriptor,Hamming-based Brute-force Matcher,then optimize the matches results, according to the PNP algorithm for realtime estimates camera pose matrix, finally, in the Android system fulfilled an augmented reality application.Experiments show that this method can effectively runs in general intelligent mobile phone, and in the light of different angles, sign changes, partial occlusions, it has a good Robustness.
\end{abstract}

\section{Introduction}

Augmented reality technique is a new technique based on virtual reality technique. With the help of computer vision technique and visualization technology, it overlaps the virtual image or other information in the real scenes which can be say by the users, for achieving sensory experience beyond reality [1]. Three-dimensional registration method is generally applied in the mobile terminal to implement augmented reality. For feature point extraction, E.Rosten proposed FAST[2] algorithm. The algorithm uses the information comparing surrounding pixels to achieve feature points. The algorithm is easy and the computation is rapid, but the disadvantage is that the extracted feature points may include feature point blocks and it is sensitive to rotation and light. The common natural feature description algorithm includes SIFT3] algorithm and SURF [4] algorithm. The algorithms have good performance, but the algorithms have the disadvantages of great complexity and large computation, which makes it difficult to be applied to the mobile terminal. The paper proposes a three-dimensional registration method for mobile platform, and the method is used to realize augmented reality on Android mobile phones. ORB feature extraction and FREAK feature description improves the real-time performance of the algorithm. Hanming distance matching algorithm is used for feature point matching, which improves feature point matching speed. Then, the matrix of the camera is achieved according to real-time estimation of PNP algorithm, and three-dimensional virtual objects are superimposed in a real scene. The experimental results indicate that the method has the advantages of great real-time performance and good robustness under different conditions.

Android is a open source operation system based on Linux kernel, and it is mainly applied to mobile equipment including smartphone and tablet personal computer. The open source feature of Android system provides free development environment for the developers, which achieves the support of manufactures and develops rapidly in recent years. Android system applies the idea of layered architecture, and it is application layer, application framework, program library, Android working hour and Linux kernel from the top layer to the bottom layer. The structure of four layers is clear, and they operated collaboratively. OpenCV is an open-source cross-platform computer vision library. It is written by $\mathrm{C} / \mathrm{C}++$ language, and it provides language interfaces such as $\mathrm{C}, \mathrm{C}++$ and Java. It supports Windows, Linux, Mac OS, IOS and Android. OpenCV includes many universal algorithms relating to image processing and computer vision. The paper implements the algorithms for three-dimensional registration with the help of OpenCV. 


\section{Augmented Reality System Implementation Scheme of Android Platform}

Problems and solutions. How to implement augmented reality system on mobile phones under the condition of ensuring real time and robustness of the algorithm is a difficulty of developing augmented reality system. The paper proposes a new three-dimensional registration method. The implementation idea is as follows. Firstly, ORB algorithm is used to extract feature points of images. Then, FREAK with good robustness is used to describe feature points, and coercive matching algorithm based on Hanming distance is used to match description subset. And the achieve match receives optimization. The relationship between template image and video frame is computed, and the pose of the camera is estimated. Lastly, the rendering model is superimposed in real scenes to implement integration of virtual and reality. The scheme of using the algorithm on Android mobile phones is shown in Figure 1. The scheme is divided into Java layer and Native layer. Two layers are connected by Android NDK, which improves the operation efficiency of the system. Java layer implements the acquisition of video frame, offline training of template images and rendering of three-dimensional virtual models. Native layer implements the algorithms relating to image processing. Two layers implement data communication by NDK programming.

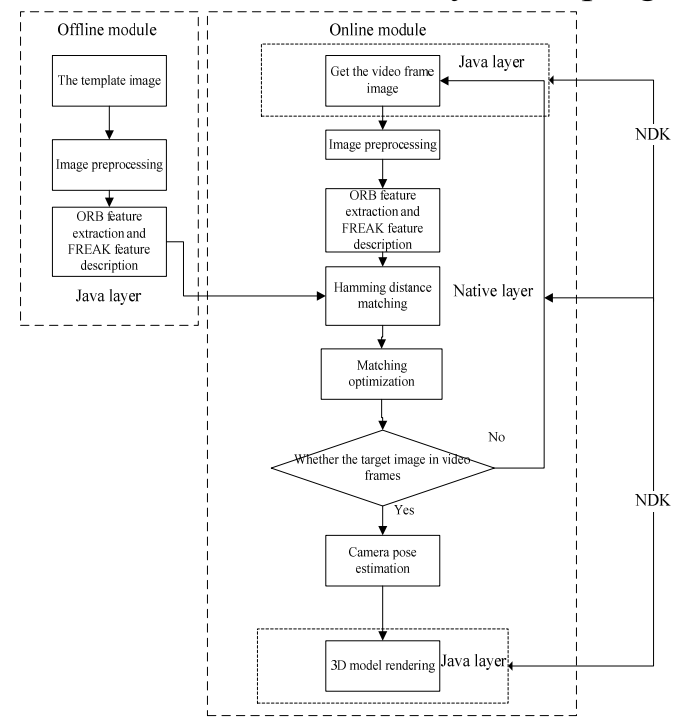

Fig. 1. Implementation of augmented reality on android platform

\section{Three-dimensional Registration Method of Augmented Reality of Android Platform}

Feature point detection. In order to save sources of mobile phones and reduce energy consumption, the paper selects ORB feature point detection algorithm with little computation and rapid speed is used for ensuring real-time performance of the system.

ORB algorithm is the improvement of FAST feature detection algorithm and BRIFF feature description operator. ORB detection algorithm uses FAST algorithm with the rapidest speed, and uses Harris algorithm to remove the edge effect.

FAST algorithm is a feature point detection algorithm of non-scale space, and it is generally used in gray-scale images. The feature points are defined as follows. When the gray level in the neighborhood of the detected pixel points is greater or less than the gray level of the point, the detection point is marked as angular point. Formula 1 is generally used to judge if the candidate pixel points are angular points,

$$
F=\sum_{0 \leq a \leq N} 2^{a} T\left(p_{a}\right)
$$

$P$ is the central pixel point, and $X$ is the neighborhood pixel point of $P$. $I(X)$ and $I(P)$ means the gray level of point $X$ and point $P$ of the image, and $t$ is the presetting threshold. The number of pixel points $\mathrm{N}$ is compared with $\mathrm{t}$. If it is greater than $\mathrm{t}$, the candidate point $\mathrm{P}$ is an angular point. 
Feature point description. For feature point description, the paper uses FREAK descriptor. Compared with traditional SIFT and SURF, FREAK has the characteristics of rapid computation and good robustness.

FREAK is a binary descriptor. The core idea is to use the sampling template imitating retinal structure for descriptor structure. Fixed sampling mode is used, and sampling templates of FREAK use different Gaussian kernels for smoothing. And the smoothing kernel of each sampling point is in direct proportional to the diameter of the circle. The nearer it is from feature center, the less Gaussian kernel, the greater the sampling density.

FREAK uses Gaussian convolution difference with correction sampling points to establish binary descriptor $\mathrm{F}$, as shown in formula 2 .

$$
F=\sum_{0 \leq a \leq N} 2^{a} T\left(p_{a}\right)
$$

$P_{a}$ is a pair of sampling points, $\mathrm{N}$ means the dimensions of descriptors, and $T\left(P_{a}\right)$ meets formula 3 .

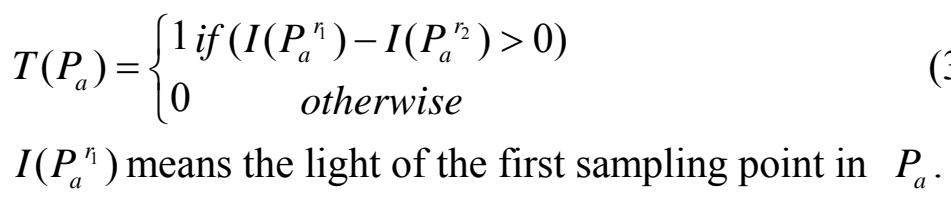

For the principal direction of computing feature points, FREAK uses the way which is similar to BRISK[10] algorithm. It computes local gradient to represent the principal direction of features. It is different from that BRISK algorithm that FREAK algorithm uses sampling domain with central symmetry.

The computation formula 4 of feature point direction is as follows.

$$
H=\frac{1}{M} \sum_{P_{0} \in G}\left(I\left(P_{0}^{r_{1}}\right)-I\left(P_{0}^{r_{2}}\right)\right) \frac{P_{0}^{r_{1}}-P_{0}^{r_{2}}}{\left\|P_{0}^{r_{1}}-P_{0}^{r_{2}}\right\|}
$$

$\mathrm{H}$ means the set of all sampling points of computing gradient. $\mathrm{M}$ is the pairs of sampling points of the set $\mathrm{H}$, and $P_{0}^{r_{1}}$ and $P_{0}^{r_{2}}$ is the two-dimensional vector of sampling center.

Compared with sampling points of BRISK, the computation of FREAK feature direction only needs 43 feature points, which not only improves the speed, but also reduces the memory.

Feature point matching. After extracting and describing feature points, the target images and video frame images need to receive feature point matching. SIFT algorithm and SURF algorithm computes Euclidean distance and uses the nearest ratio to determine matching points. The difference is that FREAK algorithm generates binary descriptor, so Hanming distance is used to compute the distance of descriptors. When the distance of two descriptors is less than the presetting threshold, it is considered as a matching pair. In order to improve matching accuracy, 16-byte Hanming distance is compared, and then the later bytes is used for accurate matching. After forcing matching of Hanming distance, it is common that there are many mismatching points, as shown in Figure 2. In order to ensure the accuracy of the matching results, the system uses cross matching for optimization. Then, RANSAC algorithm is used to eliminate mismatching point pairs to improve the accuracy of matching algorithm. The matching effect after optimization is shown in Figure 3.
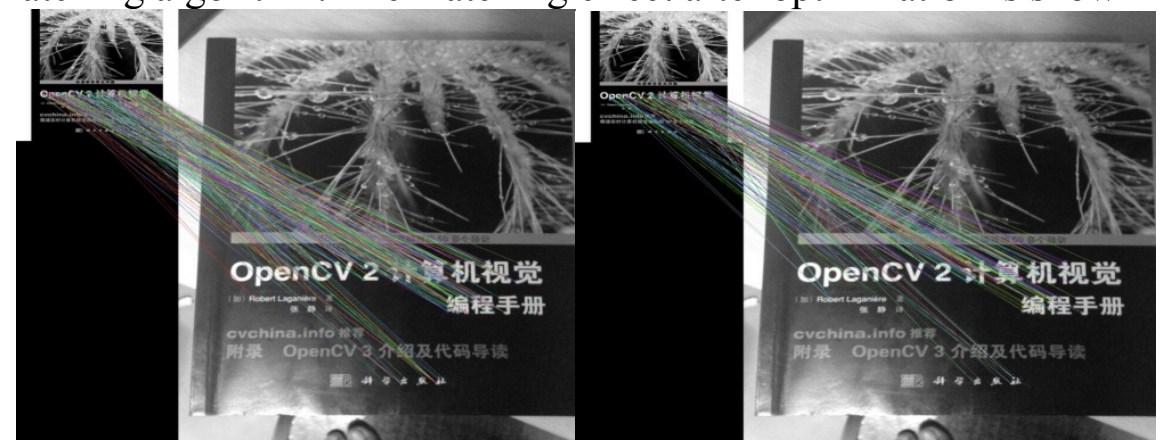

Fig. 2 matching effect before optimized

Fig. 3 matching effect afteroptimized 
The number of matching pairs and the correct matching ratio before and after optimization is shown in Table 3.1.

Tab 1 Contrast before and after optimization

\begin{tabular}{cccc}
\hline Name & $\begin{array}{c}\text { Number } \\
\text { matching }\end{array}$ & of feature point & Accuracy rate (\%) \\
\hline Before optimization & 358 & 51 \\
After optimization & 224 & 80 \\
\hline
\end{tabular}

From Table 3.1, we can see that there are many feature point matching pairs before optimization, and the accuracy rate is low. The matching pairs reduce evidently after optimization, which indicates that the mismatching pairs are eliminated after optimization, which improves the matching accuracy.

Coordinate transformation and model rendering. After achieving the matching results, the projection location and angle of the virtual images needs to be computed, which requires the transformation of different space coordinates. In order to estimate the pose of camera, there is need to know the projection equation of the camera, and the definition of scaling factor $\mathrm{S}$ is as follows.

$$
S\left(\begin{array}{c}
u \\
v \\
1
\end{array}\right)=\left(\begin{array}{ccc}
f_{x} & 0 & c_{x} \\
0 & f_{y} & c_{y} \\
0 & 0 & 1
\end{array}\right)=\left(\begin{array}{ll}
R_{3 \times 3} & T_{3 \times 1}
\end{array}\right)\left(\begin{array}{c}
x \\
y \\
z \\
1
\end{array}\right)=P\left(\begin{array}{c}
x \\
y \\
z \\
1
\end{array}\right)
$$

$f_{x}, f_{y}, c_{x}$ and $c_{y}$ means the internal parameters of the camera, $(X, Y, Z)^{T}$ is the world coordinate system, $(u, v)^{T}$ means the two-dimensional image coordinate system of world coordinate system after projection transformation. $R_{3 \times 3}$ and $T_{3 \times 1}$ means the rotation matrix and translation matrix of the camera under real environment. $\mathrm{P}$ is the projection matrix of the cameral in real environment, which can realize coordinate transformation of two-dimensional coordinate and three-dimensional space.

From formula 5, we can see that in order to get the projection matrix of the camera, the homography matrix needs to be computed according to the matching information. Then, according to the angular point of the template images, the homography matrix is used to map out the location of angular point coordinate of video streaming images. Lastly, PnP algorithm is used to get the external parameters of the camera, and three-dimensional model generated by OpenGL ES is rendered in real scene to implement three-dimensional registration, which achieves the effect of integration of virtual and reality.

\section{Experimental Verification and Result Analysis}

Algorithm real-time performance test. In order to prove the advantage of time performance, SIFT algorithm, SURF algorithm, FAST algorithm, BRISK algorithm and the method in the paper is used for feature point detection of template images. And the number of the detected feature points and the time is shown in Table 2.

Tab 2 The comparison results of different feature point detection algorithm

\begin{tabular}{cccccc}
\hline $\begin{array}{c}\text { Feature point } \\
\text { detection } \\
\text { algorithm }\end{array}$ & SIFT & SURF & FAST & BRISK & $\begin{array}{c}\text { Algorithm in } \\
\text { the paper }\end{array}$ \\
\hline $\begin{array}{c}\text { Number of } \\
\text { feature points } \\
\text { Time for }\end{array}$ & 392 & 537 & 1472 & 376 & 479 \\
detection (ms) & 736 & 33 & 186 & 64 \\
\hline
\end{tabular}


From Table 2, we can see that the detection time of ORB extraction algorithm is evidently better than that of SIFT algorithm, SURF algorithm and BRISK algorithm. The detection time of FAST algorithm is better than ORB algorithm, but it includes many useless feature points, and too many feature points may increase the time of describing feature points.

Verification of stability and robustness of algorithm. In order to verify the stability and robustness of the algorithm, the algorithm is implemented on Android cellphones. And rotation angle, distance, scale and occlusion is set. And the experimental results are shown as follows.

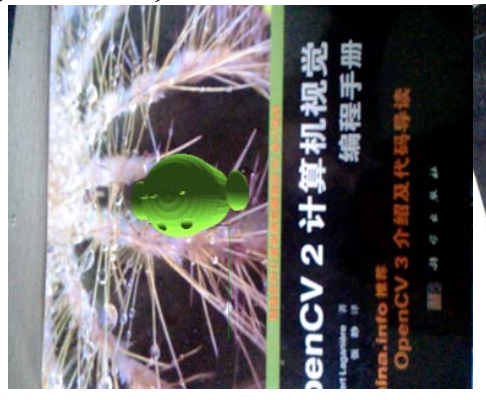

(a) angle 1

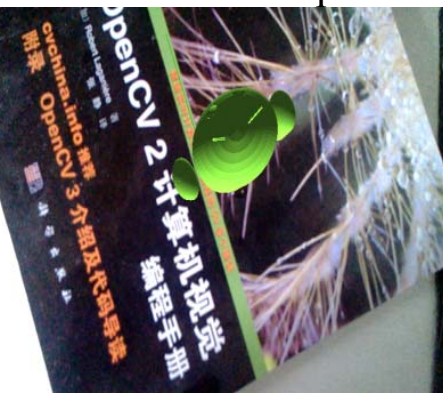

(b)angle2
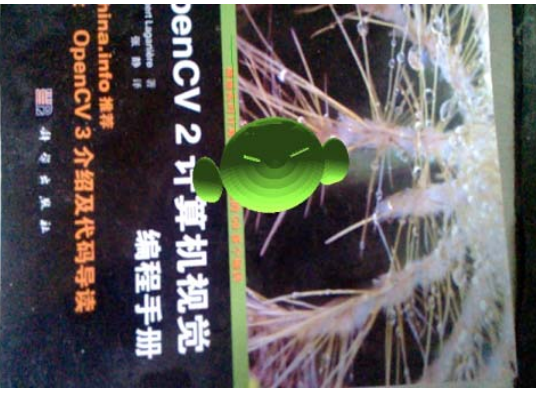

(c) angle3

Fig. 4 Registered under the effect of rotation changes

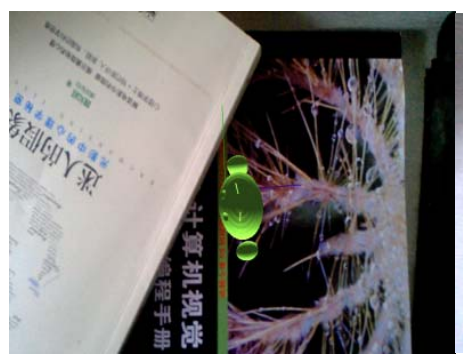

(a)the upper half is occluded

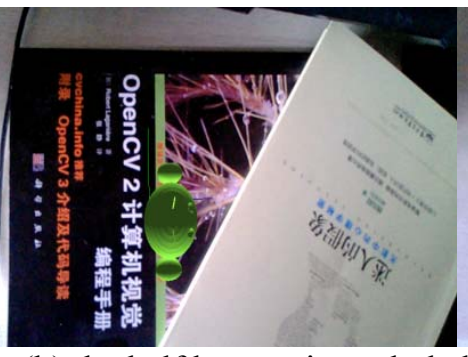

(b) the half bottom is occluded

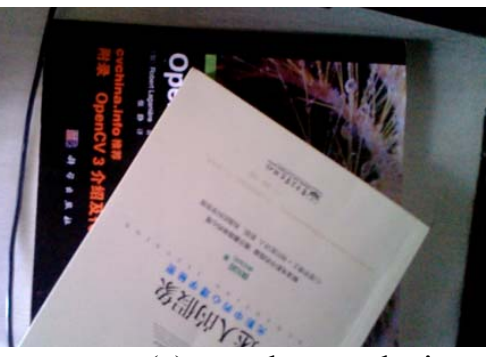

(c)complete occlusion

Fig. 5 Registered under the effect of occlusion changes

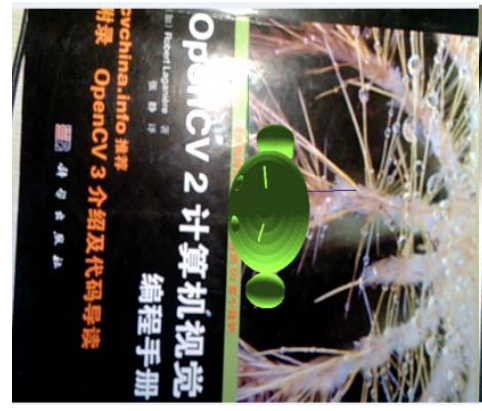

(a)strong illumination

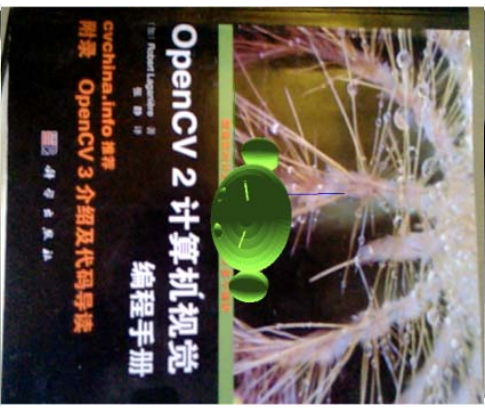

(b)normal illumination

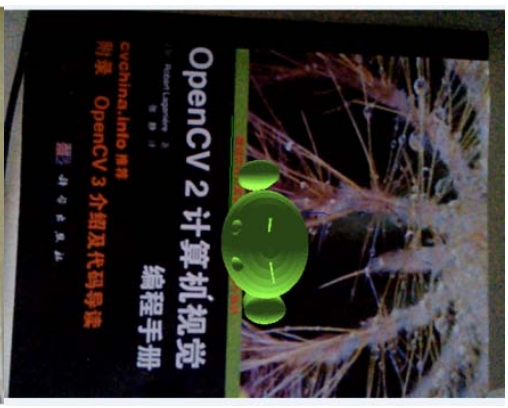

(c)weak illumination

Fig. 6 Registered under the effect of illumination changes

We can see from the experiment that the system has the characteristics that the virtual model registration is stable and there is no excursion under the conditions of rotation change, occlusion and illumination change, which verifies that the stability and robustness of the algorithm in the paper is good.

Verification of scale adaptability of the algorithm. In order to verify that the algorithm has better scale adaptability, the registration effect of the algorithm and ORB+ORB algorithm under scale change is compared, and the results are shown in Figure 7. Figure (a) is the registration effect of ORB+ ORB algorithm. When the scale changes, the model should appear in red area of the image, but virtual model has evident shift, which makes registration unstable. Figure (b) is the registration effect of the algorithm in the paper, and the model registration is stable when the scale changes. So the adaptability of the algorithm in the paper for the scale is better. 


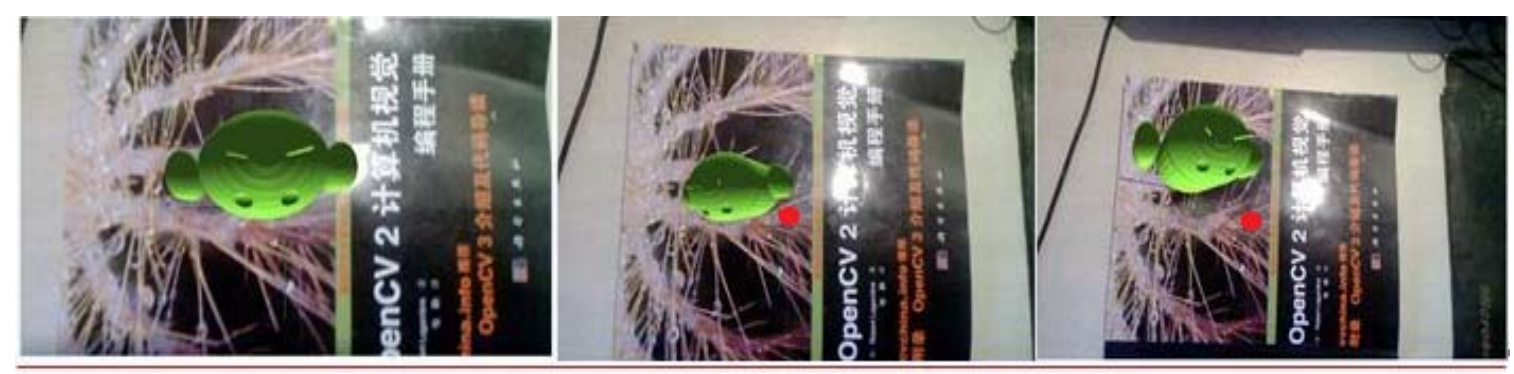

(a) Registration effect of $\mathrm{ORB}+\mathrm{ORB}$ under scale changes

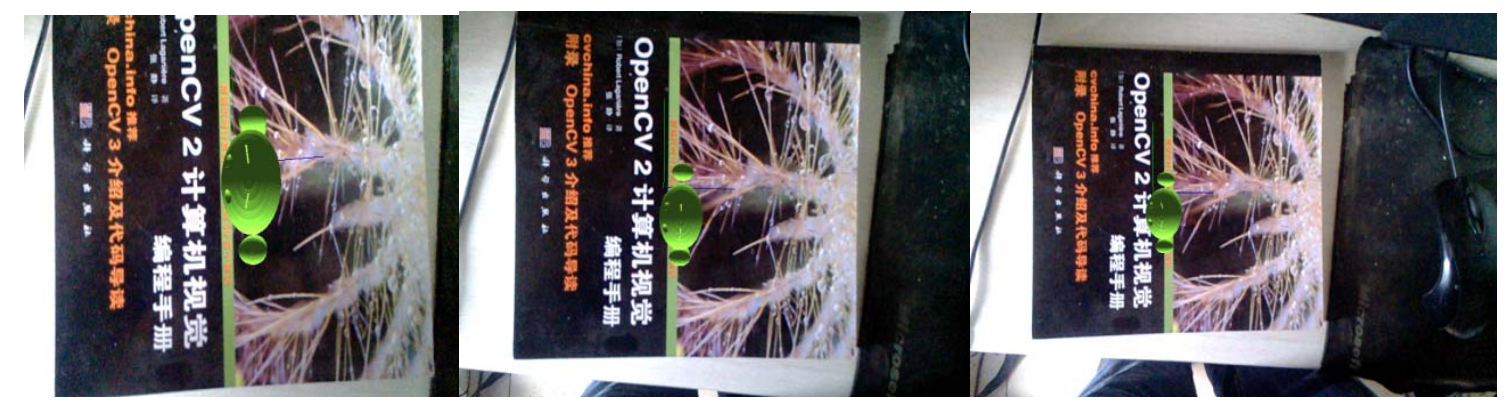

(b) Registration effect of the algorithm in the paper under scale changes

Fig. 7 Scale adaptive test

\section{Conclusions}

Augmented reality is a new technique. How to implement augmented reality technique in mobile terminal in real time has been the research hotspot in recent years. The paper proposes an augmented reality tracing registration technique based on ORB-FREAK algorithm, and applies it to Android system. The algorithm has the advantage of good real-time performance and good robustness when the environment changes. The algorithm needs to be optimized in the future, such as introducing tracing algorithm to improve real-time performance of the system.

\section{References}

[1] Sun Yuan, Chen Jing, Research on mobile augmented technique of smart phones [J], Computer Science, 2012, 39 (6A): 493-498.

[2] Rosten E,Drummeond T.Machine learning for high-speed corner detection [C]//European Conference on Computer Vision.2006:430-443.

[3] Ke Y,Sukthankar R. PCA-SIFT: A more dis-tinctive representationfor local image descriptors[C]// Proceedings ofthe 2004 IEEE Computer Society Conference on Computer Vision and Pattern Recog-nition. 2004: 506-513.

[4] Bay H, Tuytelaars T, Van Gool L. SURF: Speeded up robust features [C] // European Confe-rence on Computer Vision. 2006: 404-417.

[5] Calander M, Lepetit V, Strecha Cetal. BRIEF:Binary robust Independent elementaryfeatures [C]//European Conference on Computer Visio, Crete, Greece:ECCV, 2010:778-792.

[6] Rublee E, Rabaud V, Konolige K, et al.Orb:an efficent alternative to sift or surf [C]// proceeding of the IEEE Intetnational Conference on Computer Versi-on, Barcelona, Spain: ICCV, 2011: 2564-2571.

[7] Alahi A, Ortiz R, Vandergheynst P.FREAK:FAST Retina Keypoint [C]//Computer Version and PattenRecognition, Providence, RI, USA: CVPR, 2011:510-517. 
[8] Chen Zhixiang, Wu Liming, Gao Shiping, Mobile augmented reality tracing technique based on FAST-SURF algorithm [J], Computer and Modernization, 2013 (9): 105-108.

[9] Xiao Bin, Jiang Ge, Lin Fan, Mobile augmented reality three-dimensional registration algorithm based on ORB and KLT [J], Computer and Modernization, 2014 (3): 57-60.

[10] Stefan L, Margarrita C, Roland S.BRISK: Binary Robust invariant Scalable keypoints [C]// proceeding of the IEEE Intetnational Conference on Computer Version, Barcelona, Spain:ICCV, 2011:2548-2555. 